NOTES AND CORRESPONDENCE

\title{
A Demonstration of Long-Term Memory and Climate Predictability
}

\author{
XIUHUA ZHU* \\ Max Planck Institute for Meteorology, Hamburg, Germany \\ KLAUS FRAEDRICH \\ KlimaCampus, University of Hamburg, Hamburg, Germany \\ ZHENGYU LIU \\ University of Wisconsin-Madison, Madison, Wisconsin \\ RICHARD BLENDER \\ KlimaCampus, University of Hamburg, Hamburg, Germany
}

(Manuscript received 5 August 2009, in final form 12 March 2010)

\begin{abstract}
Climate forecast skills are evaluated for surface temperature time series at grid points of a millennium control simulation from a state-of-the-art global circulation model [ECHAM5-Max Planck Institute Ocean Model (MPI-OM)]. First, climate predictability is diagnosed in terms of potentially predictable variance fractions and the fluctuation power-law exponent (using detrended fluctuation analysis). Long-term memory (LTM) with a fluctuation exponent (or Hurst exponent) close to 0.9 occurs mainly in high-latitude oceans, which are also characterized by high potential predictability. Next, explicit prediction experiments for various time steps are conducted on a gridpoint basis using an autocorrelation predictor. In regions with LTM, prediction skills are beyond that expected from red noise persistence-exceptions occur in some areas in the southern oceans and over the Northern Hemisphere continents. Extending the predictability analysis to the fully forced simulation shows a large improvement in prediction skills.
\end{abstract}

\section{Introduction}

The predictability of a system is closely linked to its short- and long-term memory (LTM). While short-term memory is described by an exponentially decreasing autocorrelation function (ACF) with a finite integral time scale, LTM is linked to a power-law decay of the ACF of a time series, $C(t) \sim t^{-\gamma}$, with $0<\gamma<1$, and it is thus characterized by an infinite integral time scale. This LTM has been observed in globally and hemispherically averaged

\footnotetext{
* Current affiliation: KlimaCampus, University of Hamburg,
} Hamburg, Germany.

Corresponding author address: Xiuhua Zhu, KlimaCampus, Grindelberg 5, D-20144, Hamburg, Germany.

E-mail: xiuhua.zhu@zmaw.de surface air temperature (Bloomfield 1992; Gil-Alana 2005), station surface air temperature (Pelletier 1997), geopotential height at $500 \mathrm{hPa}$ (Tsonis et al. 1999), paleoclimate temperature proxies (Pelletier 1997; Huybers and Curry 2006), sea level (Barbosa et al. 2006), and many other studies; and it is characterized by enhanced low-frequency variability and has great implications for long-term forecast and extreme events (Blender et al. 2008).

Detrended fluctuation analysis (DFA) (Peng et al. 1994) is in wide use for LTM detection, which yields the fluctuation function $F(t)$ measuring fluctuations within the time series on a time scale $t$. For a power law in the power spectrum $S(f) \sim f^{-\beta}$ there is the relation $F(t) \sim$ $t^{\alpha}$, with $\beta=2 \alpha-1$ (Havlin et al. 1988), and the correlation exponent is related by $\beta=1-\gamma$. Therefore, DFA may be seen as a method performed in the time domain 
that is analogous to power spectrum analysis in the frequency domain but with a smoother appearance because of the inherent segment averaging. Note that $\alpha$ is similar to the Hurst exponent, except that DFA may also be applied to the data whose underlying statistics are nonstationary. It has been reported that surface temperature shows the following characteristics on decadal time scales (Fraedrich and Blender 2003): (i) $\alpha$ is close to 1 in some ocean basins suggesting nonstationary LTM; (ii) over continents, $\alpha$ is close to 0.5 revealing a white noise nature of the local processes; (iii) and $\alpha$ is around 0.65 within the transition zones along the coasts indicating weak LTM. The LTM in the North Atlantic can extend up to centennial time scales (Fraedrich and Blender 2003). Discrepancies in terms of $\alpha$ have been reported over the inner continents (Bunde et al. 2004; Rybski et al. 2006) and are attributed to different spatial coverage of the available data (Blender and Fraedrich 2004), whereas LTM in highlatitude oceans, particularly in the North Atlantic, remains robust and has been suggested to be associated with the state of the Atlantic meridional overturning circulation (Blender et al. 2006; Zhu et al. 2006). Loaded with high potential predictability (Boer 2000), the North Atlantic has received intensive attention as a test bed for the prediction capability of numerical climate models (e.g., Griffies and Bryan 1997; Keenlyside et al. 2008). Promising skills are achieved in the subpolar North Atlantic (see Fig. 4 from Griffies and Bryan 1997 and Fig. 1a from Keenlyside et al. 2008), where LTM is most pronounced, indicating a close relationship. In fact, key questions related to predictability, such as whether there is predictability at all and up to what time scales we can potentially make forecasts, depend critically on the existence of LTM; however, the usefulness of LTM for practical predictions has never been demonstrated. In particular, while red noise has been introduced as the paradigm in stochastic climate theory (Hasselmann 1976), LTM implies predictability beyond that expected from red noise damped persistence. Here, this additional predictability is demonstrated explicitly.

The present analysis is based on the millennium control simulation using the Community System Models (COSMOS) Earth System Model, which includes atmosphere, ocean, land surface, and biogeochemistry. By analyzing the near-surface temperature in the control simulation, we first present the spatial distribution of potentially predictable variance fraction ( $p p v f)$ as a rough approximation of predictability, followed by the spatial pattern of the DFA-derived power-law scaling exponent $\alpha$. Using a first-order autoregressive (AR1) linear predictor, we further demonstrate that, in areas with LTM, predictability is beyond that expected from red noise processes generated by a Monte Carlo method. Results from the simulation with full external forcing, including both natural forcing (solar radiation, orbital change, and volcanic forcing) and anthropogenic forcing (land-use change and greenhouse gas forcing), are also presented. This article is arranged as follows: section 2 introduces the model description; section 3 introduces the methods to describe potential predictability and memory, ppvf and DFA, respectively; and a detailed description of the forecast procedure is given in section 4 , followed by the results (section 5), and then the conclusions and discussion (section 6).

\section{Model description: Millennium simulation}

Our analyses are based on the millennium experiments using the COSMOS Atmosphere-Land-OceanBiogeochemistry Earth System Model, which consists of the atmosphere model (ECHAM5; Roeckner et al. 2003), Max Planck Institute Ocean Model (MPI-OM; Marsland et al. 2003), and modules for land vegetation, Jena Scheme for Biosphere-Atmosphere coupling in Hamburg (JSBACH; Raddatz et al. 2007), and for ocean biogeochemistry, Hamburg Model of the ocean carbon cycle (HAMOCC; Wetzel et al. 2006), which are coupled via the Ocean Atmosphere Sea Ice Soil version 3 (OASIS3) coupler. The model setup enables the interactive simulation of the carbon cycle. ECHAM5 is run at T31 resolution with 19 vertical levels, and MPIOM is at a horizontal grid spacing of about $3^{\circ}$ by $3^{\circ}$, with 40 vertically uneven-spaced levels.

The model is forced by reconstructions of (i) total solar irradiance (TSI), (ii) volcanic forcing considering aerosol optical depth (AOD) and effective radius distribution, (iii) land-use change, and (iv) anthropogenic greenhouse gases and aerosols. The TSI reconstruction is based on unpublished data by N. Krivova (2008, unpublished manuscript). The volcanic effects are taken into account in AOD units for 10-day time steps and split into 4 equal area segments $\left(30^{\circ}-90^{\circ} \mathrm{N}, 30^{\circ} \mathrm{N}-0^{\circ}, 30^{\circ} \mathrm{S}-0^{\circ}\right.$, and $\left.30^{\circ} \mathrm{S}-90^{\circ}\right)$. The growth and decay time for each eruption are calibrated and fitted to recent observations. The dataset includes information about mean particle radius evolution versus time, a feature particularly important for large eruptions (for details see Crowley et al. 2008). The land-use change is considered in terms of crop and pasture fraction, which extend back in time using a scaling dependent on population density (Pongratz et al. 2008). Here, we analyzed the unforced control integration in which all external forcings are kept constant and one experiment forced by full external forcing. The former is $2000 \mathrm{yr}$ long after a 3000-yr spinup and the latter is $1206 \mathrm{yr}$ long. The annualmean near-surface $(2 \mathrm{~m})$ air temperature field is analyzed. 


\section{Statistical methods: Potentially predictable variance fractions and DFA}

The ppvf is estimated following Boer $(2000,2004)$ and Boer and Lambert (2008) to describe potential predictability. A time series $X$ with length $N=n \times m$ is decomposed into $n$ nonoverlapping $m$-yr segments $X_{i, j}$, with $i$ labeling the $i$ th $m$-member chunk of the data, and $j$ is the location within the chunk. The variability of the time series is decomposed into signal and noise according to $X_{i, j}=\delta_{i}+\varepsilon_{i, j}$. Here, $\delta_{i}$ is the average of the $i$ th chunk, $\delta_{i}=(1 / m) \sum_{j=1}^{m} X_{i, j}$, describing the internally generated potentially predictable long time-scale component, and $\varepsilon_{i, j}$ is the deviation from $\delta_{i}, \varepsilon_{i, j}=X_{i, j}-\delta_{i}$, representing the unpredictable short noise component. Given the variance of the long time-scale component $\sigma_{\delta}^{2}$ and the total variance of the time series $\sigma^{2}$, the potentially predictable variance fraction is estimated by $\rho=\sigma_{\delta}^{2} / \sigma^{2}$ (see Boer and Lambert 2008 for details).

The ppvf $\rho$ quantifies the potentially predictable contribution at the time scale of $m$ yr. If the long-term variability signal rises above its noise counterpart with appreciable magnitude, it is presumed that this signal, most likely, results from physical processes that can be predicted given sufficient knowledge and information.

DFA has been developed to detect long-range powerlaw correlations (Peng et al. 1994) and is used to analyze time series with superimposed trends of any polynomial shape (e.g., Govindan et al. 2002; Fraedrich and Blender 2003). The method is implemented as follows: the anomalies $X_{k}^{\prime}$ are integrated to the profile, $Y_{i}=\sum_{k=1}^{i} X_{k}^{\prime}$, for $i=$ $1, \ldots, N$. The profile time series is partitioned into segments of duration $t$, where linear trends or high-order polynomials with degree $N$ are fitted (DFA-N). After the fit is removed from each segment, the variance of the residuals is computed, and the fluctuations $F(t)$ are given by the means of the variances over all segments. Higher-order DFA-N is applied when trends or higher-order polynomial growth types are present in the time series. In a typical time series obtained from a complex system, $F(t)$ obeys a power-law function $F(t) \sim t^{\alpha}$. The scaling exponent $\alpha$, obtained from the slope of the linear regression of $F(t)$ versus $t$ on a log-log scale, quantifies the long-range correlations in the time series. For stationary processes with LTM, $\alpha$ lies between white noise $(\alpha=0.5)$ and flicker noise $(\alpha \approx 1)$; and for Brownian noise, $\alpha$ is close to 1.5. It is important to note that, because $\alpha$ depends upon line fitting to the DFA fluctuation function in a log-log plot, appearances of strong periodicity will deteriorate the scaling behavior by lifting up the fluctuation function (as periodic signals appear in a power spectrum) and make it difficult to estimate $\alpha$. Therefore, in tropical regions where ENSO dominates, $\alpha$ should be interpreted with caution. To maximize the utilization of the data, we allow $30 \%$ overlapping during segment partitioning, and the maximum segment length is restricted to one-quarter of the data length - that is, 500 data points for the 2000-yr control simulation and around 300 points for the 1206-yr full forcing simulation. In this study, DFA-2 is applied, which removes linear trends in the data.

\section{Forecast procedures}

We first introduce the forecast procedure followed by an example forecast of a temperature time series to demonstrate the method.

\section{a. Forecast method and confidence test}

Forecasts are performed using an AR1 predictor at each grid point. Our aim is to forecast the $m$-yr average; thus, the data is binned and averaged over $m$ (in years), the time scale of interest. The method is described in detail as follows (take forecasts of 5-yr averages as an example):

1) The annual time series $X_{k}, k=1, \ldots, N(N=2000)$ is divided into $n=400$ chunks, each containing $m$ members, where $m$ is given by $N / n=5$; the average over each chunk forms the target time series, $\delta_{i}=$ $(1 / m) \sum_{j=1}^{m} X_{i, j}, i=1, \ldots, n$.

2) An AR1 process is fitted to the target time series $\delta_{i}$. With the derived AR1 parameter, a forecast (denoted by $*$ ) is made at each data point $\delta_{i}^{*}$.

3) Skills are evaluated by the correlation coefficients between the target time series $\delta_{i}$ and the forecast $\delta_{i}^{*}$.

4) To assess the forecast skill at longer time scales, we increase the length of $m$ and repeat steps 1-3.

As we are particularly interested to know LTM-related predictability, we will test our prediction skills against the forecast from AR1 processes generated by a Monte Carlo method by using the lag-1 autocorrelation coefficient of the original time series: at each grid point, we fit the original yearly time series $X_{k=1 \ldots N}$ to an AR1 model; with the obtained AR1 parameters, 100 red noise ensembles $(\mathrm{R} 1, \ldots, \mathrm{R} 100)$ are generated with variance equal to that of $X_{k=1, \ldots, N}$. Subsequently, steps $1-4$ are followed for each ensemble. The $95 \%$ quartile is taken as the $95 \%$ confidence level. In this manner, the demonstrated predictability should be interpreted in reference to that derived from red noise processes.

\section{b. A forecast example}

For demonstration, we present an example forecast with a temperature time series taken from the North Atlantic (the location is marked as + in Fig. 2d). The original time series is presented in Fig. 1a. It shows scaling between $10^{0.8}-10^{1.8} \mathrm{yr} \sim(6-60)$ yr with $\alpha \approx 1$ (1/f noise or flicker 

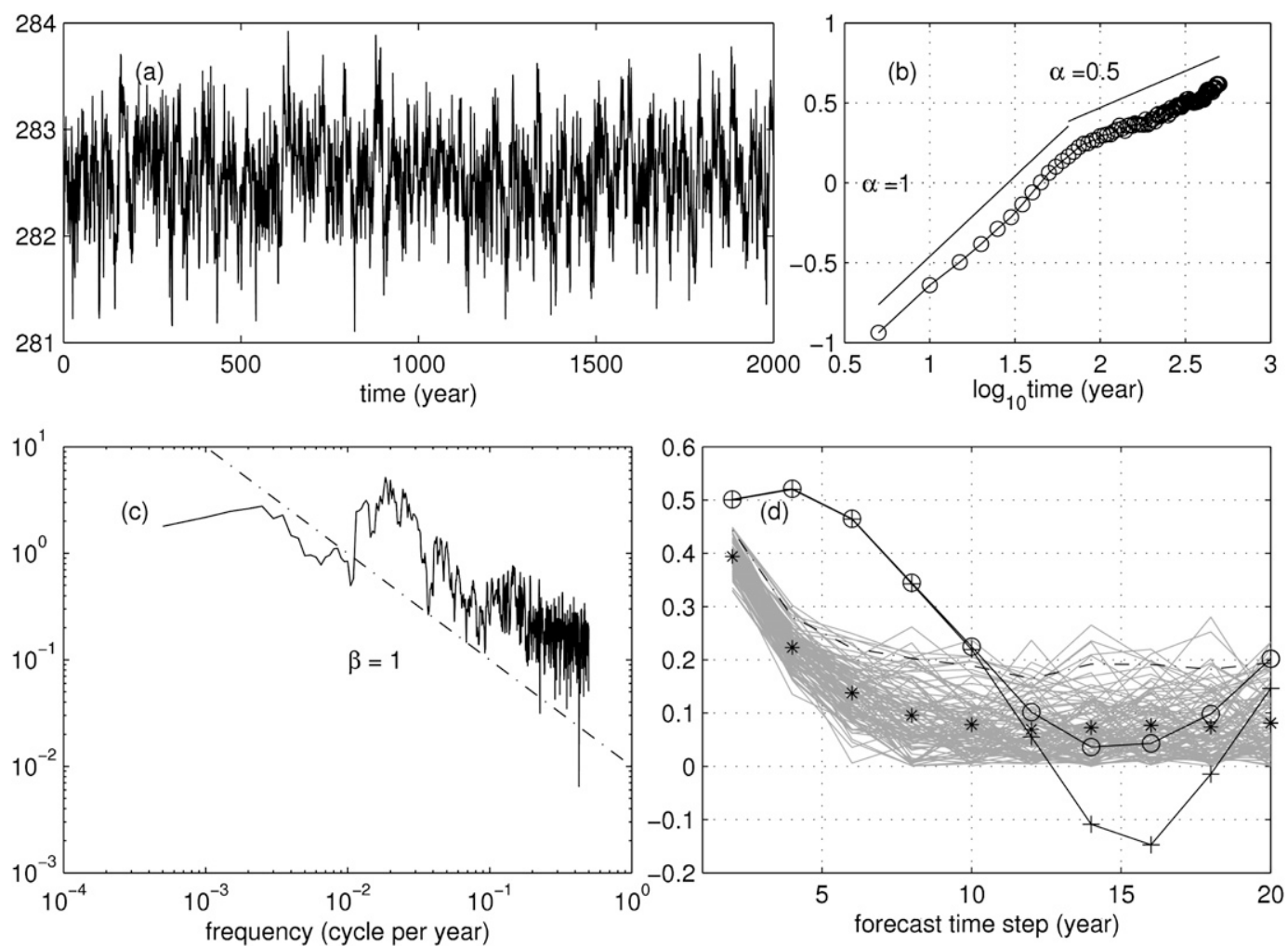

FIG. 1. An example forecast: (a) 2000-yr near-surface temperature time series (the location is marked as + in Fig. 2d); (b) DFA-2 fluctuation function; (c) power spectrum; (d) and correlation scores at 2-, 4-, 6-, .., 20-yr scales. In (b), the scaling exponent $\alpha$ is estimated between $10^{0.8}-10^{1.8} \mathrm{yr}(\sim 6-60 \mathrm{yr})$ as the slope of this function in a log-log plot. The mean of the entire time series is removed in (c), and the dashed line denotes $\beta=1$. In (d), black circled and open circled lines denote the hindcast and the leave-one-out forecast, respectively. Gray lines are hindcasts of red noise processes generated using the lag-1 autocorrelation coefficient of the original time series (Fig. 1a), and the dash-dotted line denotes the $95 \%$ confidence level. Here, “*” denotes the mean of the correlation scores of all ensembles. Hindcast results are shown in later figures if not otherwise specified.

noise) and white noise characteristics at longer time scales with $\alpha \approx 0.5$ (Fig. 1b) as supported by the power spectrum (Fig. 1c). Skillful predictions are obtained for up to 10-yr time scales (black circled line in Fig. 1d) - that is, up to this time scale, the example time series has predictability beyond that derived from pure red noise processes.

Strictly speaking, the previous result shows only a "hindcast" instead of a forecast because the AR1 parameters are derived considering all data points. To further validate the forecast method, we perform a "leave-one-out" validation; in step 2 of the forecast procedures (section $4 \mathrm{a}$ ), one point $\delta_{i}$ is not considered for estimating AR1 parameters. With the derived AR1 parameters and the knowledge of the data point one step ahead $\delta_{i-1}$, a forecast $\delta_{i}^{*}$ is performed. The forecast scores are evaluated by the correlation coefficients between $\delta_{i}$ and $\delta_{i}^{*}$, with $i=2, \ldots, n$. The leave-one-out forecast correlation score (black crossed line in Fig. 1d) is almost identical to the hindcast for up to 10-yr-average predictions and underestimated at longer time scales
(Fig. 1d). In subsequent sections, results from hindcast practices, that is, AR1 parameters derived considering all data points, are presented and named as forecast.

\section{Spatial structure of potential predictability and memory}

In this section, we present spatial characteristics of potential predictability and memory in the near-surface temperature field, followed by results from the prediction experiments at various time scales. Promising prediction skills are obtained at decadal time scales (decadal-mean average predictions) in areas with LTM, which are characterized by high potential predictability. Exceptions are found in some parts of the southern oceans.

\section{a. Potential predictability}

The ppvf $\rho$ decreases slowly with increasing time scales, with negligible values (smaller than $10 \%$ ) over continents 
(a) 5-year-average ppvf

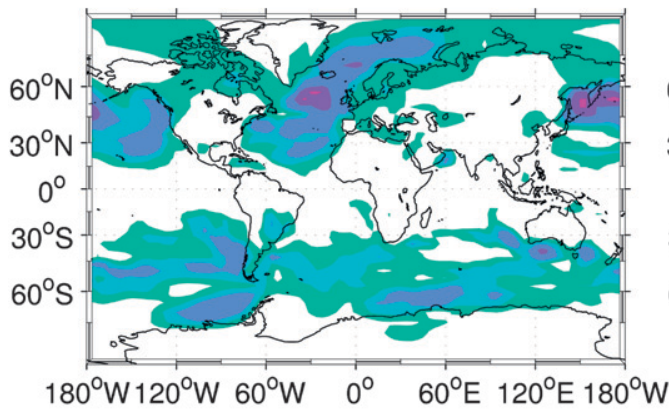

(c) 15-year-average ppvf

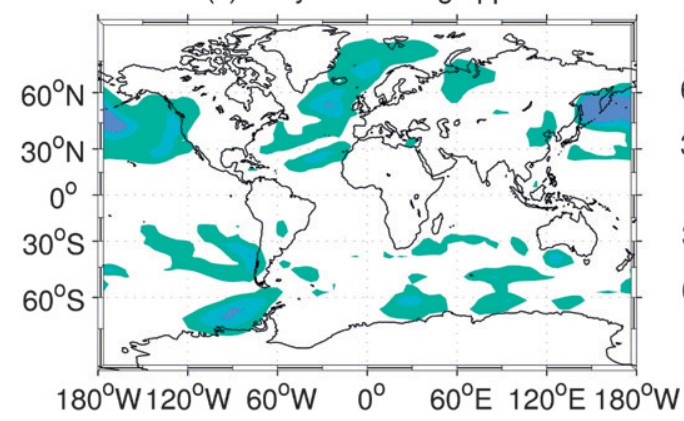

(b) 10-year-average ppvf

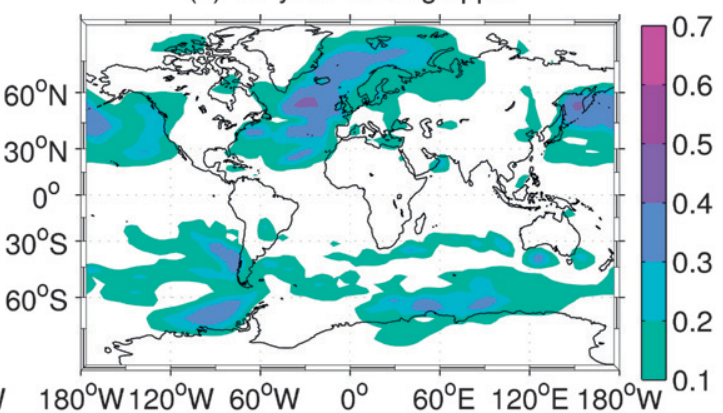

(d) DFA2 $\alpha$

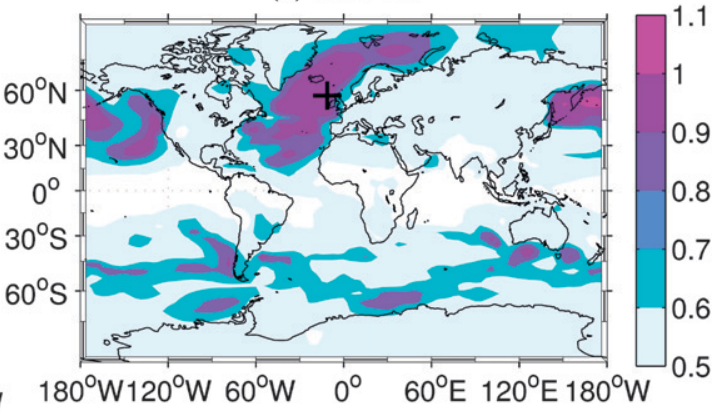

FIG. 2. (a)-(c) Ppvf $\rho$ for the 5-, 10-, and 15-yr averages (values $<0.1$ are not colored). Panels (a)-(c) share the top colorbar. (d) DFA-2 fluctuation exponent $\alpha$ for 2000-yr near-surface temperature estimated between $10^{0.8}-10^{1.8} \mathrm{yr}$ $[\sim(6-60 \mathrm{yr})]$; values $<0.5$ are not colored. The cross sign in (d) denotes the location of the selected time series analyzed in Fig. 1.

and tropical regions, and with higher values (between $20 \%$ and $60 \%$ ) in high-latitude oceans (Figs. 2a,b); some incursion into North America, the Mediterranean regions, and Eurasia is also observed, with modest loadings of $\rho$ (Fig. 2a). Considerable potential predictability is still observed on a $30-y r$ scale, with $\rho$ between $20 \%$ and $40 \%$ in the subpolar North Atlantic, the Drake Passage, the southern Indian Ocean, and the North Pacific (not shown).

\section{b. Distribution of memory}

DFA-2 fluctuation exponent $\alpha$ shows the following features: (i) over equatorial oceans, $\alpha$ is smaller than 0.5 because of the masking of the periodic ENSO; (ii) over continents and large areas in the Southern Ocean, $\alpha$ is between 0.5-0.6; (iii) in high-latitude oceans of the Northern Hemisphere-namely, in the North Atlantic, the Norwegian Sea, and the North Pacific $-\alpha$ is between 0.8 and 1, suggesting the presence of LTM; (iv) and LTM also occurs along the path of the Antarctic Circumpolar Current (ACC), with $\alpha$ between 0.6 and 0.9. This spatial pattern agrees well with previous studies (Fraedrich and Blender 2003), except that the area coverage in the Southern Ocean with LTM is smaller in this millennium run.

It is noteworthy that there is a close correspondence between the spatial patterns of the ppvf $\rho$ and the DFA fluctuation exponent $\alpha$; particularly in the high-latitude oceans, where LTM occurs with $\alpha$ between 0.8 and 1 (up to $60 \mathrm{yr}$ ) and the potentially predictable component accounts for around $20 \%$ of the total variance on a $15-\mathrm{yr}$ scale.

\section{c. Predictability}

Prediction results from the control simulation are noted as follows: (i) high predictability is obtained in highlatitude oceans where LTM is observed, more distinctly represented in the Northern Hemisphere, in particular the subpolar Atlantic and the subpolar Pacific, where the predictability is still significant at 10 -yr time scales (Fig. 3b); (ii) forecast scores decrease with increasing time scales, for example, in both the subpolar Atlantic and the subpolar Pacific, the AR1 predictor loses skills for 15-yraverage predictions (Fig. 3c); (iii) and one noteworthy area is near $50^{\circ} \mathrm{S}$ in the South Atlantic, where high scores persist even on a 15-yr time scale (Figs. 3a-c). As an exception to the previously mentioned close correspondence between LTM and predictability, skillful predictions are also obtained in 1) the equatorial Pacific and Indian Oceans at the 5-yr scale and 2) in North America, the Mediterranean region, and central Asia at up to the 10-yr scale (Figs. 3a-b), although there is no LTM detected (Fig. 2d).

The entire analysis has also been applied to the simulation forced by the full forcing, which covers the years 
(a) 5-year-average

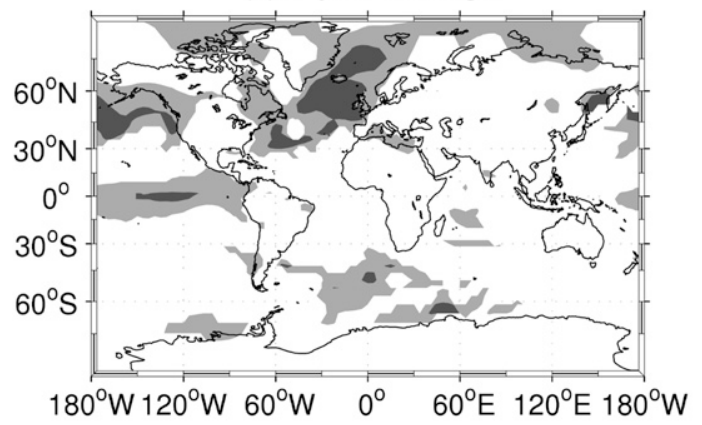

(c) 15-year-average

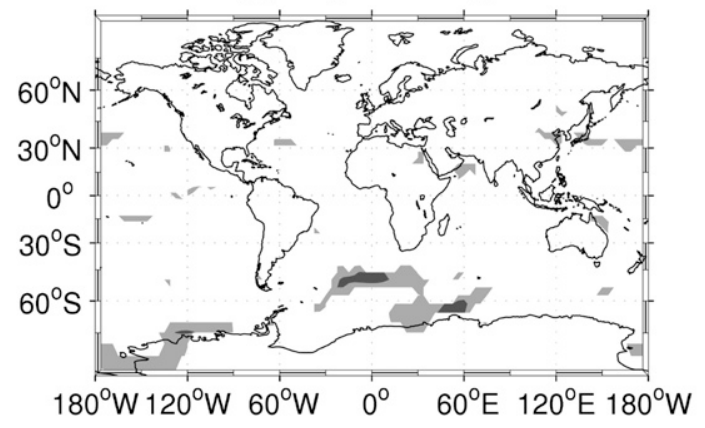

(b) 10-year-average

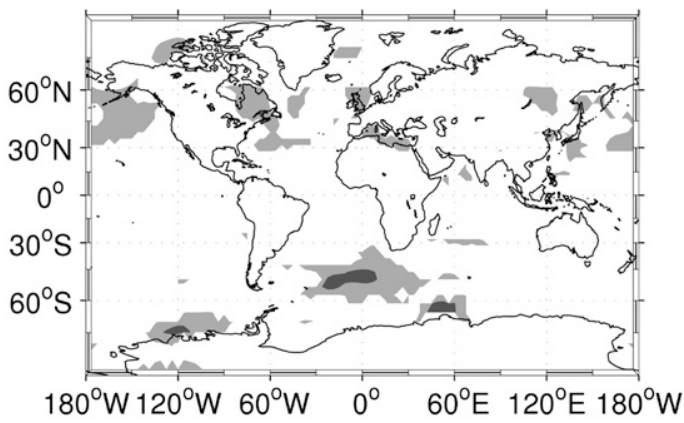

FIG. 3. Correlation scores of forecasts for (a) 5-, (b) 10-, and (c) 15-yr-average near-surface temperature from the control simulation. Values passing the $95 \%$ confidence test are shown.

800-2005. Large-scale improvement in prediction is obtained in the subpolar Atlantic, the Mediterranean region, the tropical Indian Ocean, and central Eurasia (Fig. 4). Interestingly, in most of the previously mentioned regions and in the western tropical Atlantic, skills remain significant at the 15-yr time scale (Fig. 4c). Meanwhile, reduced skills are observed in the equatorial Pacific at the 5- and 10 -yr time scale, but additional skills are obtained in the tropical Atlantic and persist up to 10 -yr time scales (Fig. 4b).

To investigate whether the anthropogenic warming during the recent decades contributes to the improvement in predictability shown in Fig. 4, a linear warming trend, derived from the last $30 \mathrm{yr}$, is removed at each grid point prior to prediction practices. The prediction scores are shown in Fig. 5. The improvement of the predictions skills in the western tropical Atlantic, the Mediterranean region, the tropical Indian Ocean, and central Eurasia in the full forcing experiment (Fig. 4) remains (Fig. 5); therefore, they cannot be directly attributed to the warming. On the other hand, when the linear warming trend is removed, prediction skills are reduced in the eastern tropical Pacific, the subtropical South Atlantic $\left(10^{\circ}-45^{\circ} \mathrm{S}\right)$, the western tropical Indian Ocean, and central Asia at 5-yr time scales (Fig. 5), implying that in the previously mentioned regions, the recent warming tends to enhance predictability.

\section{Conclusions and discussion}

In this article, based on a state-of-the-art millennium control simulation, we have demonstrated the existence of LTM in the near-surface temperature field in highlatitude oceans, which is most pronounced in the North Atlantic and North Pacific with DFA-2 fluctuation exponent $\alpha$ close to 1 -these regions bear also high potential predictability at time scales of more than a decade. Forecast experiments based on an AR1 linear predictor are employed at different time scales. We find that in areas with LTM, the prediction skills are beyond those expected from pure red noises.

High loadings of the potentially predictable variance fractions occur where LTM is found (Figs. 2a-d), particularly over high-latitude oceans where the DFA fluctuation exponent $\alpha$ is close to 1 . This close correspondence indicates that high potential predictability in the highlatitude oceans may be attributed to the LTM, which is likely of an oceanic origin (Zhu et al. 2006). It implies that improvement in prediction skills may be more attainable in areas with LTM, provided more sophisticated prediction methods are available, than in places showing no memory-for example, over the inner continents. Note that in comparison to previous studies from multimodel approaches (see Fig. 4 from Boer and Lambert 2008), 
(a) 5-year-average

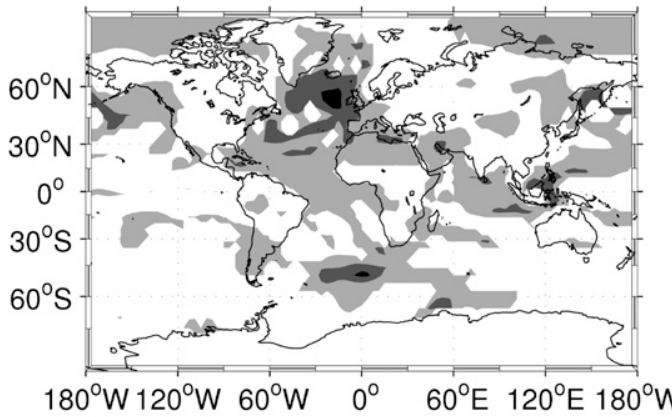

(c) 15-year-average

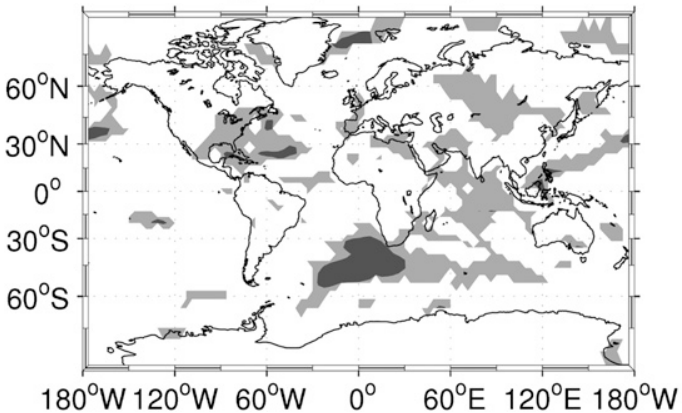

FIG. 4. As in Fig. 3, but for the 1206-yr full forcing experiment. (b) 10-year-average
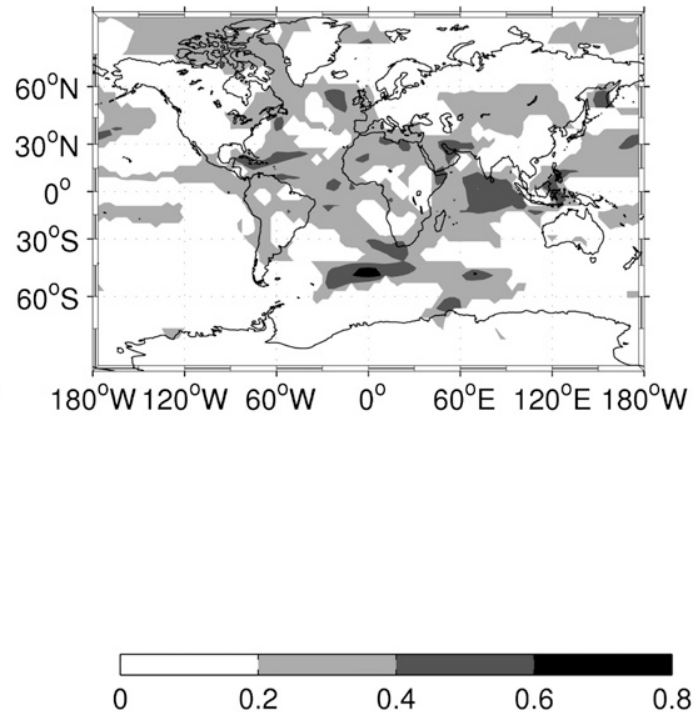

potential long time predictability is less pronounced in the southern Atlantic in the current millennium simulation.

Despite the close tie between LTM and predictability in the high-latitude oceans of the Northern Hemisphere, exceptions are found in the southern Indian Ocean and the southern Pacific, where the DFA-2 fluctuation exponent $\alpha$ shows values between 0.6 and 0.9 , which indicates the presence of LTM (Fig. 2d), and potential (a) 5-year-average

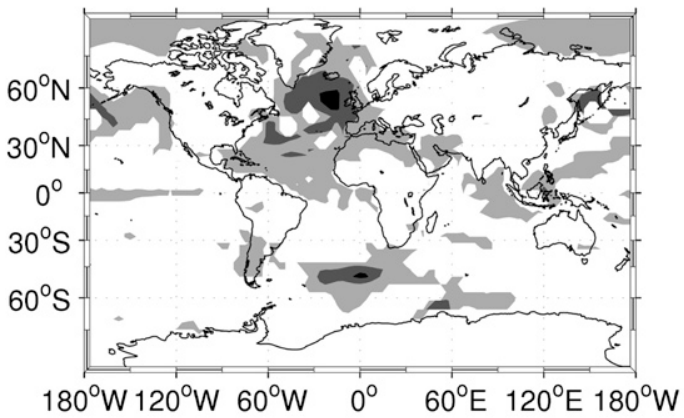

(c) 15-year-average

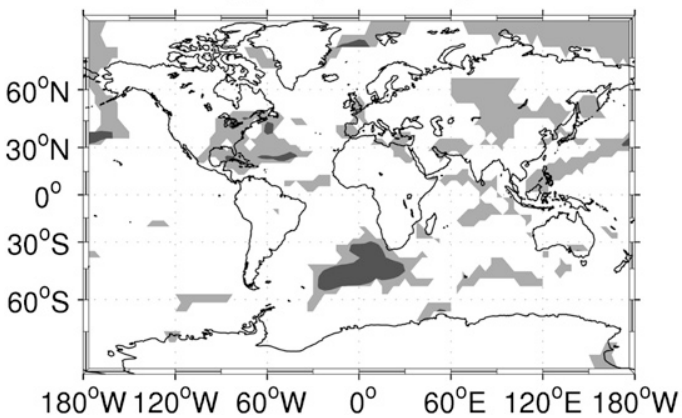

(b) 10-year-average

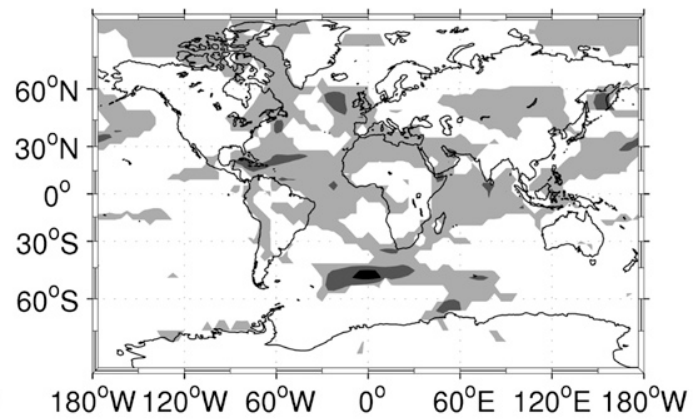

$180^{\circ} \mathrm{W} 120^{\circ} \mathrm{W} 60^{\circ} \mathrm{W} \quad 0^{\circ} \quad 60^{\circ} \mathrm{E} 120^{\circ} \mathrm{E} 180^{\circ} \mathrm{W}$

FIG. 5. As in Fig. 4, but with the linear warming trend (derived from the last $30 \mathrm{yr}$ ) removed. 
predictability $\rho$ accounts for up to $40 \%$ of the total variance at 10-yr intervals (Fig. 2b), but the AR1 linear predicator shows almost no skills (Figs. 3a-c). A closer look reveals that, in these two regions, scaling with $\alpha$ close to 1 extends only up to $25 \mathrm{yr}$ (instead of $\sim 60 \mathrm{yr}$, Fig. $2 \mathrm{~d})$. With such a short scaling, the LTM time series can be simulated by red noise processes as well, so that both can hardly be distinguished in terms of predictability. Another exception is observed over some parts of North America, the Mediterranean region, and northeast Asia, where prediction skills are obtained even at $10-\mathrm{yr}$ time scales (Fig. 3b): while in these cases $\alpha$ is between $0.5-0.6$ (Fig. 2d) suggesting a white noise nature, or if any, weak memory, considerable ppvf loadings are observed in these areas (Figs. 2a,b). In this case, DFA-revealed LTM and the potential predictability supplement each other in locating regions where prediction practices may gain success.

Another noteworthy feature is that in the central southern Atlantic, a patch of area shows high predictability (correlations scores between 0.2 and 0.6) persistent through all the time scales considered (Figs. 3a-c). This high predictability results from the local strong decadal and bicentennial fluctuations, which enhance the predictable component by AR1 processes but deteriorate the scaling properties in DFA analysis. Their origin is not clear.

It is important to stress that the prediction skill demonstrated in this article defines only the predictability beyond that expected from an AR1 damping process; therefore, if not significant, it does not mean "lost skill," instead, it means that the LTM does not play a role significantly different from that expected from a red noise process. On the other hand, even if it is beyond the predictability of red noise processes, care should be taken when the correlation skills are considered for future climate forecast - even in regions where LTM dominatesbecause, for instance, a correlation score of 0.2 means that the predictable variability explains only $0.2 \times 0.2=$ $4 \%$ of the total variance.

When extending the forecast experiment to the simulation with full forcing, predictability is improved considerably, in particular, in the western tropical Atlantic, the Mediterranean region, the tropical Indian Ocean, and central Eurasia (Fig. 4). Furthermore, the improvement remains within most of the previously mentioned areas when the warming influence is linearly removed (Fig. 5); therefore, it is less likely contributed by the anthropogenic warming. As it is still under debate whether, and to what extent, individual forcing may contribute to scaling properties in climate variables (e.g., Blender and Fraedrich 2004; Vyushin et al. 2004a,b), it is of special importance in the near future to explore the possible effect of each individual forcing on LTM and predictability.
Acknowledgments. Thanks are due to all involved, including the millennium project in the Max Planck Institute for Meteorology and the German Computer center (DKRZ) where the simulations were performed. We wish to thank Dr. Katja Lohmann and Frank Sielmann for their help with the interpretation of the results. The authors also appreciate the helpful suggestions from two anonymous reviewers. Work was supported by the Max Planck Fellow Group "Climate System LongTerm Variability."

\section{REFERENCES}

Barbosa, S. M., M. J. Fernandes, and M. E. Silva, 2006: Long-range dependence in North Atlantic sea level. Physica A, 371, $725-731$.

Blender, R., and K. Fraedrich, 2004: Comment on "Volcanic forcing improves atmosphere-ocean coupled general circulation model scaling performance" by D. Vyushin, I. Zhidkov, S. Havlin, A. Bunde, and S. Brenner. Geophys. Res. Lett., 31, L22213, doi:10.1029/2004GL020797.

_, , and B. Hunt, 2006: Millennial climate variability: GCM-simulation and Greenland ice cores. Geophys. Res. Lett., 33, L04710, doi:10.1029/2005GL024919.

,$--\frac{1}{\longrightarrow}$, and F. Sienz, 2008: Extreme event return times in longterm memory processes near 1/f. Nonlinear Processes Geophys., 15, 557-565.

Bloomfield, P., 1992: Trends in global temperature. Climatic Change, 21, 1-16.

Boer, G. J., 2000: A study of atmosphere-ocean predictability on long time scales. Climate Dyn., 16, 469-477.

- 2004: Long time-scale potential predictability in an ensemble of coupled climate models. Climate Dyn., 23, 29-44.

—, and S. J. Lambert, 2008: Multi-model decadal potential predictability of precipitation and temperature. Geophys. Res. Lett., 35, L05706, doi:10.1029/2008GL033234.

Bunde, A., J. F. Eichner, S. Havlin, E. Koscielny-Bunde, H. J. Schellnhuber, and D. Vyushin, 2004: Comment on "Scaling of Atmosphere and Ocean Temperature Correlations in Observations and Climate Models." Phys. Rev. Lett., 92, 039801, doi:10.1103/PhysRevLett.92.039801.

Crowley, T. J., G. Zielinski, B. Vinther, R. Udisti, K. Kreutz, J. Cole-Dai, and E. Castellano, 2008: Volcanism and the Little Ice Age. PAGES News, Vol. 16, No. 2, PAGES International Project Office, 3012 Bern, Switzerland, 22-23.

Fraedrich, K., and R. Blender, 2003: Scaling of atmosphere and ocean temperature correlations in observations and climate models. Phys. Rev. Lett., 90, 108501, doi:10.1103/ PhysRevLett.90.108501.

Gil-Alana, L. A., 2005: Statistical modeling of the temperatures in the Northern Hemisphere using fractional integration techniques. J. Climate, 18, 5357-5369.

Govindan, R. B., D. Vyushin, A. Bunde, S. Brenner, S. Havlin, and H.-J. Schellnhuber, 2002: Global climate models violate scaling of the observed atmospheric variability. Phys. Rev. Lett., 89, 028501, doi:10.1103/PhysRevLett.89.028501.

Griffies, S. M., and K. Bryan, 1997: Predictability of North Atlantic multidecadal climate variability. Science, 275, 181-184.

Hasselmann, K., 1976: Stochastic climate models. Part I. Theory. Tellus, 28, 463-485. 
Havlin, S., R. Blumberg Selinger, M. Schwartz, H. E. Stanley, and A. Bunde, 1988: Random multiplicative processes and transport in structures with correlated spatial disorder. Phys. Rev. Lett., 61, 1438-1441.

Huybers, P., and W. Curry, 2006: Links between annual, Milankovitch, and continuum temperature variability. Nature, 441, 329-332.

Keenlyside, N. S., M. Latif, J. Jungclaus, L. Kornblueh, and E. Roeckner, 2008: Advancing decadal-scale climate prediction in the North Atlantic sector. Nature, 453, 84-88.

Marsland, S., H. Haak, J. Jungclaus, M. Latif, and F. Röske, 2003: The Max-Planck-Institute global ocean/sea ice model with orthogonal curvilinear coordinates. Ocean Modell., 5, 91-127.

Pelletier, J., 1997: Analysis and modeling of the natural variability of climate. J. Climate, 10, 1331-1342.

Peng, C.-K., S. V. Buldyrev, S. Havlin, M. Simons, H. E. Stanley, and A. L. Goldberger, 1994: Mosaic organization of DNA nucleotides. Phys. Rev., 49, 1685-1689.

Pongratz, J., C. H. Reick, T. Raddatz, and M. Claussen, 2008: A global land cover reconstruction AD 800 to 1992-Technical description. Max Planck Institute for Meteorology Rep. on Earth System Science 51, 72 pp.

Raddatz, T. J., and Coauthors, 2007: Will the tropical land biosphere dominate the climate-carbon cycle feedback during the 21 st century? Climate Dyn., 29, 565-574.
Roeckner, E., and Coauthors, 2003: The atmospheric general circulation model ECHAM5. Part I: Model description. Max Planck Institute for Meteorology Rep. 349, 127 pp.

Rybski, D., A. Bunde, S. Havlin, and H. Von Storch, 2006: Longterm persistence in climate and the detection problem. Geophys. Res. Lett., 33, L06718, doi:10.1029/2005GL025591.

Tsonis, A. A., P. J. Roeber, and J. B. Elsnerm, 1999: Long-range correlations in the extratropical atmospheric circulation: Origins and implications. J. Climate, 12, 1534-1541.

Vyushin, D., I. Zhidkov, S. Havlin, A. Bunde, and S. Brenner, 2004a: Forcing Improves atmosphere-ocean couple general circulation model scaling performance. Geophys. Res. Lett., 31, L10206, doi:10.1029/2004GL019499. $-,-, \ldots,-$, and,$- 2004 \mathrm{~b}$ : Reply to comment by R. Blender and K. Fraedrich on "Volcanic forcing improves atmosphere-ocean coupled general circulation model scaling performance." Geophys. Res. Lett., 31, L22210, doi:10.1029/ 2004 GL021155.

Wetzel, P., E. Maier-Reimer, N. Keenlyside, M. Botzet, M. Latif, and J. H. Jungclaus, 2006: Effects of ocean biology on the penetrative radiation in a coupled climate model. J. Climate, 19, 3973-3987.

Zhu, X., K. Fraedrich, and R. Blender, 2006: Variability regimes of simulated Atlantic MOC. Geophys. Res. Lett., 33, L21603, doi:10.1029/2006GL027291. 